\title{
Scale-free ferroelectricity induced by flat phonon bands in $\mathrm{HfO}_{2}$
}

\author{
Hyun-Jae Lee', Minseong Lee', Kyoungjun Lee², Jinhyeong Jo', Hyemi Yang1, Yungyeom Kim¹, Seung Chul Chae², Umesh Waghmare², Jun \\ Hee Lee ${ }^{*}$ \\ 1School of Energy and Chemical Engineering, Ulsan National Institute of Science and Technology (UNIST), Ulsan, 44919, Republic of Korea. 2Department of Physics \\ Education, Seoul National University, Seoul 08826, Korea. ${ }^{3}$ Theoretical Sciences Unit, Jawaharlal Nehru Centre for Advanced Scientific Research, Jakkur, Bangalore, \\ 560064, India.
}

*Corresponding author. Email: junhee@unist.ac.kr

Discovery of robust yet reversibly switchable electric dipoles at reduced dimensions is critical in advancing nanoelectronics devices. Energy bands flat in momentum space generate robust localized states that are activated independently of each other. We determined flat bands exist and induce robust yet independently switchable dipoles exhibiting a unique ferroelectricity in $\mathrm{HfO}_{2}$. Flat polar phonon bands in $\mathrm{HfO}_{2} \mathrm{Cause}$ extreme localization of electric dipoles within its irreducible half-unit-cell-widths $(\sim 3 \AA)$. Contrary to conventional ferroelectrics with spread dipoles, those intrinsically localized dipoles are stable against extrinsic effects such as domain walls, surface exposure, and even down-to-angstrom-scale miniaturization. Moreover, the sub-nm-scale dipoles are individually switchable without creating any domain-wall energy cost. This offers unexpected opportunities for ultimately-dense unit-cell-by-unit-cell ferroelectric switching devices directly integrable into silicon technology.

Ferroelectricity arises from spontaneous ordering of electric dipoles in a crystal which is reversibly switched to opposite directions under an applied electric field. A ferroelectric oxide $\mathrm{HfO}_{2}$ recently emerged as an interesting material because of its robust electric dipoles at nanometers thicknesses and the ability to directly integrate into silicon devices $(1-3)$. The switchability of electric dipoles in $\mathrm{HfO}_{2}$, a fluorite structure, is expected to be different from that in $\mathrm{ABO}_{3}$ perovskite-structure oxides (4) as hinted by its large coercive field $(5,6)$ and slow domain propagation (7). Unfortunately, the underlying reasons for the stable ferroelectricity and distinct switchability of $\mathrm{HfO}_{2}$ at an atomic level are poorly understood. The relationship between structure and ferroelectric properties of $\mathrm{HfO}_{2}$ is crucial for their use in advanced nanoelectronic devices like non-volatile memories and low-power logic (2).

We reveal that ferroelectric $\mathrm{HfO}_{2}$ possess switchability that is robust even down to irreducible, sub-nano-scale dimensions. This behavior is due to the flat phonon bands intrinsic to the material. While flat bands of electrons, photons, and magnons are known to cause exotic phenomena such as electron lattice (8), graphene superconductivity (9), photon(10) and magnon-localization (11), flat bands of polar phonons and their consequences in ferroelectrics are not well understood. The emergence of flat phonon bands in $\mathrm{HfO}_{2}$ provides a missing link to extend those exotic phenomena to ferroelectrics.

We used first-principles calculations to discover flat bands of polar phonons and consequent localized dipoles, which induces a scale-free ferroelectric order in $\mathrm{HfO}_{2}$. This order contains a lateral array of vertically-aligned polar layers separated by nonpolar spacer layers that are each of half unitcell width $(\sim 2.5 \AA)$. The presence of the spacers in $\mathrm{HfO}_{2}$ laterally localize the vertical dipoles within its half-unit-widths in a step-like manner from one polar layer to the next. Contrary to conventional ferroelectrics whose spread dipoles fade away below critical nano-dimensions $(4,12-15)$, the localized dipoles stable and switchable down to sub-nm scale allow storage of bits in angstrom-size lateral domains without costing any domain-wall formation energy. Vanishingly zero interactions between the ferroelectric dipoles, evidenced by flat bands in $\mathrm{HfO}_{2}$, explain the unusual phenomena of its large coercive field $(5,6)$ compared to conventional ferroelectrics (16-21) and extremely slow domain propagation (7). $\mathrm{As}^{\mathrm{HfO}_{2}}$ is already integrated into Si-technology, fabrication of ultimately dense memories could be accomplished by exploiting its irreducible unit-cell-scale switchability.

To determine the origin of unusual structural features of the orthorhombic phase of $\mathrm{HfO}_{2}\left(P c a 2_{1}\right)$, we analyzed the sequence of symmetry lowering steps starting with the cubic $F m \overline{3} m$ structure of $\mathrm{HfO}_{2}$, known to be stable above $2870 \mathrm{~K}$ (22). Upon cooling, it transforms into tetragonal $\mathrm{P}_{4_{2}} / \mathrm{nmc}$ phase at $2870 \mathrm{~K}$, and then to monoclinic phase at $T=2000$ $\mathrm{K}$. In contrast, the ferroelectric orthorhombic phase is stabilized in thin films at room temperature (I). Phonon spectrum of the high temperature cubic phase (Fig. 1A) reveals the dominant phonon instability with $X_{2}^{\prime}$ symmetry at $\omega=-i 228$ 
$\mathrm{cm}^{-1}$, which involves anti-parallel $\mathrm{x}$-displacements of neighboring oxygen atoms in yz plane (Fig. 1B). The cubic structure transforms into a tetragonal structure through the condensation of an $X_{2}^{\prime}$ phonon with zero net polarization. Among the four phonons condensing in the transformation from tetragonal to orthorhombic (fig. S1), we focus on (i) the $\Gamma_{15}^{z}$ phonon with all oxygen atoms moving along $z$-direction (Fig. 1C) generating a uniform polarization, and (ii) the cell-doubling antipolar phonon $Y_{5}^{z}$, where oxygen atoms in neighboring $\mathrm{xz}$ planes move along the z-axis in an antiparallel manner (Fig. 1D) providing A-type ordering with zero net polarization. Similar to strained $\mathrm{ZrO}_{2}$ (23), ferroelectricity of $\mathrm{HfO}_{2}$ is improperly caused by the nonlinear interaction of stable $\Gamma_{15}^{2}$ and $X_{5}$ phonons with the primarily instability of $X_{2}^{\prime}$ phonon.

An unusual aspect of the ferroelectric order originates from polar $\Gamma_{15}^{z}$ and anti-polar $Y_{5}^{z}$ phonons condensing with exactly identical amplitudes, which generate a dipolar partitioning into two types (24) of alternating atomically thin layers (Fig. 1E). The first type is the spacer with zero zdisplacements of oxygen atoms and the second is the ferroelectric layer with parallel z-displacements of its oxygen atoms. Thus, spacers are dead layers that screen the elastic interaction between the ferroelectric active layers. Experimental evidence for these layers can be seen in a study employing transmission electron microscopy (25), which named them as minor and major layers, respectively. The structural characteristics of the spacer layers is discussed in fig. S2.

Natural dipolar partitioning in orthorhombic $\mathrm{HfO}_{2}$ has spectacular consequences to its polarization domain structure, contrary to that of perovskite ferroelectric $\mathrm{PbTiO}_{3}$. As the local polarization vanishes in the spacer layer even in uniformly polarized $\mathrm{HfO}_{2}$, it inherently hosts a domain wall of vanishing thickness between oppositely polarized $\left(180^{\circ}\right)$ domains (Fig. 2A). Such a domain wall is essentially strain-free (Fig. 2, $\varepsilon_{i}$ where $i=x, y$, and $z$, fig. S3), with little change in the local structure and supporting unsuppressed bulk polarization in its neighborhood. This should lead us to expect a high energy cost of a sharp domain wall in $\mathrm{HfO}_{2}$ due to the $g|\vec{\nabla} \times \vec{P}|^{2}$ term. But $g \sim 0$ is evident in its flat band of polar phonons (Fig. 2B, supplementary text S1) and makes a sharp domain wall feasible. This flatness of the polar bands results in a phonon velocity of nearly zero (Fig. 2D). The origin of the flatness is discussed with a spring model in fig. S4. The $\Gamma$ point phonon in the flat band of the lowest frequency (black line in Fig. 2B) involves atomic displacements of all the modes condensed during cubic to orthorhombic transition, and that of higher frequency (red line in Fig. 2B) involves polar and antipolar modes. Physically, the elastic interaction between ferroelectric domains is screened by the spacer layer.
With contribution mostly from dipole-dipole interactions, the domain wall energy of $\mathrm{HfO}_{2}$ is weakly negative $\left(\sim-18 \mathrm{~mJ} / \mathrm{m}^{2}\right.$, supplementary text, section 2). In contrast, a domain wall separating the $180^{\circ}$ polar domains in $\mathrm{PbTiO}_{3}$ is diffuse, with a width of a few unit cells and the polarization is suppressed significantly in its neighborhood (Fig. 2C). This difference is because some of the polar atomic displacements are shared between adjacent domains in $\mathrm{PbTiO}_{3}$. The parameter $g$ is sizeable, as evident in its dispersed polar phonons (26) and a finite phonon velocity in $\mathrm{PbTiO}_{3}$ (Fig. 2D).

We seek to establish the stability and switchability of halfa-unit-cell-wide polar domain sandwiched between the spacers by simulating reversal of its local polarization (Fig. 3A and fig. S6). The 2D layer with flipped polarization has a robust stability, with a large energy barrier of $1.34 \mathrm{eV}$ that prevents it from switching back to the uniformly polarized state (Fig. 3B). The switching of polarization in the adjacent layer (Fig. 3B) results in a domain consisting of two ferroelectric layers sandwiching a spacer, following a path with a comparable energy barrier of $1.38 \mathrm{eV}$. Low dependence of domain wall energy on the width of polar domains reveals weak inter-domain wall interaction as expected from the flat bands. In contrast, our simulation of a single-unit-cell-wide domain in $\mathrm{PbTiO}_{3}$ (Fig. 3C) has substantially reduced polarization at the diffused domain wall, and its marginal stability is evident in its tendency to expand spontaneously (with a small energy barrier of $0.024 \mathrm{eV}$ ) to domains of larger width (Fig. 3D). Despite the strongly knit three-dimensional crystal structure of $\mathrm{HfO}_{2}$, our results establish that it consists of weakly interacting 2D polar layers, allowing stable and switchable ferroelectric domains at the ultimate limit of width $(2.7 \AA)$.

We provide a possible explanation of the puzzling observation that the coercive field of polarization switching observed in $\mathrm{HfO}_{2}$ is unusually large and even comparable to the activation field $\left(E_{\mathrm{a}}\right)$. The coercive field in conventional ferroelectrics is typically a tenth of the activation field (Fig. 4A) (16), as polarization switching occurs by nucleation and the growth of ferroelectric domains of reversed polarization. Due to such collective behavior, $E_{\mathrm{c}}$ is generally reduced by a factor proportional to the width of the domain wall (27). In $\mathrm{HfO}_{2}$, domain walls are vanishingly thin and the resulting reduction in $E_{\mathrm{c}}$ (Fig. 4A) is marginal. With weakly interacting domain walls and zero group velocity of the relevant polar modes ( $g \sim 0$, by the flat bands of $\mathrm{HfO}_{2}$ ), domain walls do not propagate efficiently and can move only by hopping over a large energy barrier (Fig. 3B), suggesting that their sluggish motion observed experimentally could be an intrinsic property $(7,28)$. In contrast, a domain wall in $\mathrm{PbTiO}_{3}$ encounters a much smaller energy barrier of $0.024 \mathrm{eV}$ (Fig. 3D) and its motion leads to rapid expansion of its polar domain.

We demonstrate the scale-free nature of polarization switching in $\mathrm{HfO}_{2}$ by comparing reversal of uniform and local 
polarization (Fig. 4B). Energetics of local and uniform polarization switching in $\mathrm{HfO}_{2}$ are strikingly similar. Flipping the polarization of a single layer is nearly energetically equivalent (per layer) to flipping the polarization of all layers. In contrast, reversal of local polarization in a single unit cell wide region is energetically forbidden in $\mathrm{PbTiO}_{3}$. Total energy along the polarization reversal in consecutive $2 \mathrm{D}$ polar domains in $\mathrm{HfO}_{2}$ is a periodic function of the number of unitcell-width domains switched (Fig. 4C). The equal multi-stability and identical switching barriers show absolutely scalefree behavior that can be labeled by the integer (number of unit cells) and be a basis for a multi-level device whose number of states are similar to the number of lateral unit-cells. In contrast to this multi-state polar nature in $\mathrm{HfO}_{2}$, only bi-stability in $\mathrm{PbTiO}_{3}$ is evident from its uniformly polarized states $\left( \pm P_{0}\right)$ which are more stable than the rest of the intermediate polar states (Fig. 4C, bottom).

To establish the intrinsic size limit on ferroelectricity in $\mathrm{HfO}_{2}$, we simulated Hf-terminated slabs (Fig. 4D) perpendicular to (i) (010) axis with in-plane polarization, and (ii) (001) axis with out-of-plane polarization. The polarization in a ferroelectric layer of (010) slabs survives in a scale-free manner down to 1.5-unit-cell thickness with spacers acting as natural protective coatings. The polarization retains its bulk value in (001) slabs down to single-unit-cell thickness, as expected from the improper nature of fluorite ferroelectricity (29). Thus, the intrinsic lateral and perpendicular size limits on ferroelectric order in $\mathrm{HfO}_{2}$ films are 0.75 and $0.51 \mathrm{~nm}$, respectively (fig. S5). Robust ferroelectric order appears to exist down to $1 \mathrm{~nm}$ thickness, as recently reported (30), which verifies one of our predictions. Now the storage size limitation is only from the electrode and transistor used to interface with it for readout operations.

As $\mathrm{HfO}_{2}$ is already compatible in Si-electronics, our discovery of independently switchable polar layers could provide opportunities to realize ultra-dense and low cost FeRAM or FeFET for memory or logic device applications (figs. S11 and S12). In addition, possibility of unit-cell-by-unit-cell dipolar control provides different opportunities for deterministic multilevel switching (figs. S6, S7, and S12), ultimately down to the angstrom scale.

\section{REFERENCES AND NOTES}

1. T. Böscke, J. Müller, D. Bräuhaus, U. Schröder, U. Böttger, Ferroelectricity in hafnium oxide thin films. Appl. Phys. Lett. 99, 102903 (2011). doi:10.1063/1.3634052

2. T. Mikolajick, S. Slesazeck, M. H. Park, U. Schroeder, Ferroelectric hafnium oxide for ferroelectric random-access memories and ferroelectric field-effect transistors. MRS Bull. 43, 340-346 (2018). doi:10.1557/mrs.2018.92

3. M.-K. Kim, J.-S. Lee, Ferroelectric analog synaptic transistors. Nano Lett. 19, 20442050 (2019). doi:10.1021/acs. nanolett $9 b 00180$ Medline

4. C. H. Ahn, K. M. Rabe, J.-M. Triscone, Ferroelectricity at the nanoscale: Local polarization in oxide thin films and heterostructures. Science 303, 488-491 (2004). doi:10.1126/science.1092508 Medline
5. D. Zhou, Y. Guan, M. M. Vopson, J. Xu, H. Liang, F. Cao, X. Dong, J. Mueller, T. Schenk, U. Schroeder, Electric field and temperature scaling of polarization reversal in silicon doped hafnium oxide ferroelectric thin films. Acta Mater. 99, 240-246 (2015). doi:10.1016/i.actamat.2015.07.035

6. C. Alessandri, P. Pandey, A. Abusleme, A. Seabaugh, Switching dynamics of ferroelectric Zr-Doped HfO2. IEEE Electron Device Lett. 39, 1780-1783 (2018). doi:10.1109/LED.2018.2872124

7. P. Buragohain, C. Richter, T. Schenk, H. Lu, T. Mikolajick, U. Schroeder, A. Gruverman, Nanoscopic studies of domain structure dynamics in ferroelectric La: HfO2 capacitors. Appl. Phys. Lett. 112, 222901(2018). doi:10.1063/1.5030562

8. Z. Li, J. Zhuang, L. Wang, H. Feng, Q. Gao, X. Xu, W. Hao, X. Wang, C. Zhang, K. Wu, S. X. Dou, L. Chen, Z. Hu, Y. Du, Realization of flat band with possible nontrivial topology in electronic Kagome lattice. Sci. Adv. 4, eaau4511 (2018). doi:10.1126/sciadv. aau4511 Medline

9. Y. Cao, V. Fatemi, S. Fang, K. Watanabe, T. Taniguchi, E. Kaxiras, P. Jarillo-Herrero, Unconventional superconductivity in magic-angle graphene superlattices. Nature 556, 43-50 (2018). doi:10.1038/nature26160 Medline

10. S. Mukherjee, A. Spracklen, D. Choudhury, N. Goldman, P. Öhberg, E. Andersson, R. R. Thomson, Observation of a localized flat-band state in a photonic Lieb lattice. Phys. Rev. Lett. 114, 245504 (2015). doi:10.1103/PhysRevLett.114.245504 Medline

11. J. Schulenburg, A. Honecker, J. Schnack, J. Richter, H.-J. Schmidt, Macroscopic magnetization jumps due to independent magnons in frustrated quantum spin lattices. Phys. Rev. Lett. 88, 167207 (2002). doi:10.1103/PhysRevLett.88.167207 Medline

12. M.-W. Chu, I. Szafraniak, R. Scholz, C. Harnagea, D. Hesse, M. Alexe, U. Gösele, Impact of misfit dislocations on the polarization instability of epitaxial nanostructured ferroelectric perovskites. Nat. Mater. 3, 87-90 (2004). doi:10.1038/nmat1057 Medline

13. J. F. Ihlefeld, D. T. Harris, R. Keech, J. L. Jones, J.-P. Maria, S. Trolier-McKinstry, Scaling effects in perovskite ferroelectrics: Fundamental limits and processstructure-property relations. J. Am. Ceram. Soc. 99, 2537-2557 (2016). doi:10.1111/iace.14387

14. Y. G. Wang, W. L. Zhong, P. L. Zhang, Lateral size effects on cells in ferroelectric films. Phys. Rev. B Condens. Matter 51, 17235-17238 (1995) doi:10.1103/PhysRevB.51.17235 Medline

15. D. D. Fong, G. B. Stephenson, S. K. Streiffer, J. A. Eastman, O. Auciello, P. H. Fuoss, C. Thompson, Ferroelectricity in ultrathin perovskite films. Science 304, 16501653 (2004). doi:10.1126/science.1098252 Medline

16. H. Wieder, Activation field and coercivity of ferroelectric barium titanate. J. Appl. Phys. 28, 367-369 (1957). doi:10.1063/1.1722749

17. V. Nagarajan, I. G. Jenkins, S. P. Alpay, H. Li, S. Aggarwal, L. Salamanca-Riba, A. L. Roytburd, R. Ramesh, Thickness dependence of structural and electrical properties in epitaxial lead zirconate titanate films. J. Appl. Phys. 86, 595-602 (1999). doi:10.1063/1.370772

18. J. Son, C. Park, S.-K. Kim, Y.-H. Shin, Writing ferroelectric domain bits on the $\mathrm{PbZr}_{0.48} \mathrm{Ti}_{0.52} \mathrm{O}_{3}$ thin film. J. Appl. Phys. 104, 064101 (2008). doi:10.1063/1.2978220

19. D. Pantel, Y.-H. Chu, L. W. Martin, R. Ramesh, D. Hesse, M. Alexe, Switching kinetics in epitaxial $\mathrm{BiFeO}_{3}$ thin films. J. Appl. Phys. 107, 084111 (2010). doi:10.1063/1.3392884

20. D. Fu, H. Taniguchi, M. Itoh, S. Mori, $\mathrm{Pb}\left(\mathrm{Mg}_{1 / 3} \mathrm{Nb}_{2 / 3}\right) \mathrm{O}_{3}$ (PMN) Relaxor: Dipole Glass or Nano-Domain Ferroelectric?, in Advances in Ferroelectrics, vol. 3, A. PeláizBarranco, Ed. (2012).

21. W.-H. Kim, S. M. Yoon, J. Y. Son, Ferroelectric domain wall motion in epitaxial $\mathrm{PbTiO}_{3}$ and $\mathrm{BiFeO}_{3}$ thin films. Mater. Lett. 124, 47-49 (2014). doi:10.1016/i.matlet.2014.03.069

22. R. Terki, G. Bertrand, H. Aourag, C. Coddet, Cubic-to-tetragonal phase transition of HfO2 from computational study. Mater. Lett. 62, 1484-1486 (2008). doi:10.1016/i.matlet.2007.09.006

23. S. E. Reyes-Lillo, K. F. Garrity, K. M. Rabe, Antiferroelectricity in thin-film $\mathrm{ZrO}_{2}$ from first principles. Phys. Rev. B Condens. Matter Mater. Phys. 90, 140103 (2014) doi:10.1103/PhysRevB.90.140103 
24. R. Materlik, C. Künneth, A. Kersch, The origin of ferroelectricity in $\mathrm{Hf}_{1-x} \mathrm{Zr}_{x} \mathrm{O}_{2}$ : A computational investigation and a surface energy model. J. Appl. Phys. 117, 134109 (2015). doi:10.1063/1.4916707

25. E. D. Grimley, T. Schenk, T. Mikolajick, U. Schroeder, J. M. LeBeau, Atomic structure of domain and interphase boundaries in ferroelectric $\mathrm{HfO}_{2}$. Adv. Mater. Interfaces 5, 1701258 (2018). doi:10.1002/admi.201701258

26. N. Choudhury, E. J. Walter, A. I. Kolesnikov, C.-K. Loong, Large phonon band gap in $\mathrm{SrTiO}_{3}$ and the vibrational signatures of ferroelectricity in $\mathrm{ATiO}_{3}$ perovskites: First-principles lattice dynamics and inelastic neutron scattering. Phys. Rev. B Condens. Matter Mater. Phys. 77, 134111(2008). doi:10.1103/PhysRevB.77.134111

27. S. Choudhury, Y. Li, N. Odagawa, A. Vasudevarao, L. Tian, P. Capek, V. Dierolf, A. N. Morozovska, E. A. Eliseev, S. Kalinin, Y. Cho, L. Chen, V. Gopalan, The influence of 180 ferroelectric domain wall width on the threshold field for wall motion. $J$. Appl. Phys. 104, 084107 (2008). doi:10.1063/1.3000459

28. H. Mulaosmanovic, J. Ocker, S. Müller, U. Schroeder, J. Müller, P. Polakowski, S. Flachowsky, R. van Bentum, T. Mikolajick, S. Slesazeck, Switching kinetics in nanoscale hafnium oxide based ferroelectric field-effect transistors. ACS Appl. Mater. Interfaces 9, 3792-3798 (2017). doi:10.1021/acsami.6b13866 Medline

29. N. Sai, C. J. Fennie, A. A. Demkov, Absence of critical thickness in an ultrathin improper ferroelectric film. Phys. Rev. Lett. 102, 107601 (2009). doi:10.1103/PhysRevLett.102.107601 Medline

30. S. S. Cheema, D. Kwon, N. Shanker, R. Dos Reis, S.-L. Hsu, J. Xiao, H. Zhang, R. Wagner, A. Datar, M. R. McCarter, C. R. Serrao, A. K. Yadav, G. Karbasian, C.-H. Hsu, A. J. Tan, L.-C. Wang, V. Thakare, X. Zhang, A. Mehta, E. Karapetrova, R. V. Chopdekar, P. Shafer, E. Arenholz, C. Hu, R. Proksch, R. Ramesh, J. Ciston, S. Salahuddin, Enhanced ferroelectricity in ultrathin films grown directly on silicon. Nature 580, 478-482 (2020). doi:10.1038/s41586-020-2208-x Medline

31. G. Kresse, J. Furthmüller, Efficiency of ab-initio total energy calculations for metals and semiconductors using a plane-wave basis set. Comput. Mater. Sci. 6, 15-50 (1996). doi:10.1016/0927-0256(96)00008-0

32. G. Kresse, J. Hafner, Ab initio molecular dynamics for liquid metals. Phys. Rev. B Condens. Matter 47, 558-561(1993). doi:10.1103/PhysRevB.47.558 Medline

33. G. Kresse, J. Furthmüller, Efficient iterative schemes for ab initio total-energy calculations using a plane-wave basis set. Phys. Rev. B Condens. Matter 54 , 11169-11186 (1996). doi:10.1103/PhysRevB.54.11169 Medline

34. G. Kresse, D. Joubert, From ultrasoft pseudopotentials to the projector augmented-wave method. Phys. Rev. B Condens. Matter Mater. Phys. 59, 17581775 (1999). doi:10.1103/PhysRevB.59.1758

35. P. E. Blöchl, Projector augmented-wave method. Phys. Rev. B Condens. Matter 50 , 17953-17979 (1994). doi:10.1103/PhysRevB.50.17953 Medline

36. J. P. Perdew, K. Burke, M. Ernzerhof, Generalized gradient approximation made simple. Phys. Rev. Lett. 77, 3865-3868 (1996). doi:10.1103/PhysRevLett.77.3865 Medline

37. H. J. Monkhorst, J. D. Pack, Special points for Brillouin-zone integrations. Phys. Rev. B13, 5188-5192 (1976). doi:10.1103/PhysRevB.13.5188

38. J. Müller, T. S. Böscke, U. Schröder, S. Mueller, D. Bräuhaus, U. Böttger, L. Frey, T. Mikolajick, Ferroelectricity in simple binary $\mathrm{ZrO}_{2}$ and $\mathrm{HfO}_{2}$. Nano Lett. 12, 43184323 (2012). doi:10.1021/nl302049k Medline

39. R. D. King-Smith, D. Vanderbilt, Theory of polarization of crystalline solids. Phys. Rev. B Condens. Matter 47, 1651-1654 (1993). doi:10.1103/PhysRevB.47.1651 Medline

40. D. Vanderbilt, R. D. King-Smith, Electric polarization as a bulk quantity and its relation to surface charge. Phys. Rev. B Condens. Matter 48, 4442-4455 (1993) doi:10.1103/PhysRevB.48.4442 Medline

41. S. Mueller, C. Adelmann, A. Singh, S. Van Elshocht, U. Schroeder, T. Mikolajick, Ferroelectricity in Gd-doped $\mathrm{HfO}_{2}$ thin films. ECS J. Solid State Sci. Technol. 1 , N123 (2012). doi:10.1149/2.002301iss

42. S. Mueller, J. Mueller, A. Singh, S. Riedel, J. Sundqvist, U. Schroeder, T. Mikolajick, Incipient ferroelectricity in Al-doped $\mathrm{HfO}_{2}$ thin films. Adv. Funct. Mater. 22, 24122417 (2012). doi:10.1002/adfm.201103119

43. P. D. Lomenzo, P. Zhao, Q. Takmeel, S. Moghaddam, T. Nishida, M. Nelson, C. M. Fancher, E. D. Grimley, X. Sang, J. M. LeBeau, J. L. Jones, Ferroelectric phenomena in Si-doped $\mathrm{HfO}_{2}$ thin films with TiN and Ir electrodes. J. Vac. Sci. Technol. 32, 03D123 (2014). doi:10.1116/1.4873323
44. Y. Zhang, J. Sun, J. P. Perdew, X. Wu, Comparative first-principles studies of prototypical ferroelectric materials by LDA, GGA, and SCAN meta-GGA. Phys. Rev. B 96, 035143 (2017). doi:10.1103/PhysRevB.96.035143

45. D. Sheppard, P. Xiao, W. Chemelewski, D. D. Johnson, G. Henkelman, A generalized solid-state nudged elastic band method. J. Chem. Phys. 136, 074103 (2012) doi:10.1063/1.3684549 Medline

46. K. Momma, F. Izumi, VESTA3 for three-dimensional visualization of crystal, volumetric and morphology data. J. Appl. Cryst. 44, 1272-1276 (2011) doi:10.1107/S0021889811038970

47. U. Waghmare, K. Rabe, Ab initio statistical mechanics of the ferroelectric phase transition in $\mathrm{PbTiO}_{3}$. Phys. Rev. B Condens. Matter 55, 6161-6173 (1997) doi:10.1103/PhysRevB.55.6161

48. S. Kumar, K. Hembram, U. V. Waghmare, Intrinsic buckling strength of graphene: First-principles density functional theory calculations. Phys. Rev. B Condens. Matter Mater. Phys. 82, 115411(2010). doi:10.1103/PhysRevB.82.115411

49. R. Batra, T. D. Huan, J. L. Jones, G. Rossetti Jr., R. Ramprasad, Factors favoring ferroelectricity in hafnia: A first-principles computational study. J. Phys. Chem. C 121, 4139-4145 (2017). doi:10.1021/acs.jpcc.6b11972

50. Y. Cao, V. Fatemi, A. Demir, S. Fang, S. L. Tomarken, J. Y. Luo, J. D. SanchezYamagishi, K. Watanabe, T. Taniguchi, E. Kaxiras, R. C. Ashoori, P. Jarillo-Herrero, Correlated insulator behaviour at half-filling in magic-angle graphene superlattices. Nature 556, 80-84 (2018). do:10.1038/nature26154 Medline

51. T. Böscke, S. Teichert, D. Bräuhaus, J. Müller, U. Schröder, U. Böttger, T. Mikolajick, Phase transitions in ferroelectric silicon doped hafnium oxide. Appl. Phys. Lett. 99, 112904 (2011). doi:10.1063/1.3636434

52. U. Schroeder, C. Richter, M. H. Park, T. Schenk, M. Pešić, M. Hoffmann, F. P. G. Fengler, D. Pohl, B. Rellinghaus, C. Zhou, C.-C. Chung, J. L. Jones, T. Mikolaijick, Lanthanum-doped hafnium oxide: A robust ferroelectric material. Inorg. Chem. 57, 2752-2765 (2018). doi:10.1021/acs.inorgchem.7b03149 Medline

53. T. Mittmann, M. Materano, P. D. Lomenzo, M. H. Park, I. Stolichnov, M. Cavalieri, C. Zhou, C.-C. Chung, J. L. Jones, T. Szyjka, M. Müller, A. Kersch, T. Mikolajick, U. Schroeder, Origin of ferroelectric phase in undoped $\mathrm{HfO}_{2}$ films deposited by sputtering. Adv. Mater. Interfaces 6, 1900042 (2019). doi:10.1002/admi.201900042

54. C. Künneth, R. Materlik, M. Falkowski, A. Kersch, Impact of four-valent doping on the crystallographic phase formation for ferroelectric $\mathrm{HfO}_{2}$ from first-principles: Implications for ferroelectric memory and energy-related applications. ACS Appl. Nano Mater. 1, 254-264 (2017). doi:10.1021/acsanm.7b00124

55. R. Batra, T. D. Huan, G. A. Rossetti Jr., R. Ramprasad, Dopants promoting ferroelectricity in Hafnia: Insights from A comprehensive chemical space exploration. Chem. Mater. 29, 9102-9109 doi:10.1021/acs.chemmater.7b02835

56. M. Falkowski, C. Künneth, R. Materlik, A. Kersch, Unexpectedly large energy variations from dopant interactions in ferroelectric $\mathrm{HfO} 2$ from high-throughput ab initio calculations. npj Computational Mater. 4, 73 (2018). doi:10.1038/s41524018-0133-4

57. L. Xu, T. Nishimura, S. Shibayama, T. Yajima, S. Migita, A. Toriumi, Kinetic pathway of the ferroelectric phase formation in doped $\mathrm{HfO}_{2}$ films. J. Appl. Phys. 122, 124104 (2017). doi:10.1063/1.5003918

58. U. Schroeder, E. Yurchuk, J. Müller, D. Martin, T. Schenk, P. Polakowski, C. Adelmann, M. I. Popovici, S. V. Kalinin, T. Mikolajick, Impact of different dopants on the switching properties of ferroelectric hafniumoxide. Jpn. J. Appl. Phys. 53 (8S1), 08LE02 (2014). doi:10.7567/JJAP.53.08LE02

59. C. Richter, T. Schenk, M. H. Park, F. A. Tscharntke, E. D. Grimley, J. M. LeBeau, C Zhou, C. M. Fancher, J. L. Jones, T. Mikolajick, U. Schroeder, Si doped hafnium oxide-A "fragile" ferroelectric system. Adv. Electron. Mater. 3, 1700131 (2017). doi:10.1002/aelm.201700131

60. M. Hoffmann, T. Schenk, M. Pešić, U. Schroeder, T. Mikolajick, Insights into antiferroelectrics from first-order reversal curves. Appl. Phys. Lett. 111, 182902 (2017). doi:10.1063/1.5003612

61. S. Clima, D. J. Wouters, C. Adelmann, T. Schenk, U. Schroeder, M. Jurczak, G. Pourtois, Identification of the ferroelectric switching process and dopant dependent switching properties in orthorhombic $\mathrm{HfO}_{2}$ : A first principles insight Appl. Phys. Lett. 104, 092906 (2014). doi:10.1063/1.4867975 
62. T. D. Huan, V. Sharma, G. A. Rossetti Jr., R. Ramprasad, Pathways towards ferroelectricity in hafnia. Phys. Rev. B Condens. Matter Mater. Phys. 90, 064111 (2014). doi:10.1103/PhysRevB.90.064111

63. T. Maeda, B. Magyari-Kope, Y. Nishi, in 2017 IEEE International Memory Workshop (IMW). (IEEE, 2017), pp. 1-4.

64. M. Hoffmann, M. Pešić, S. Slesazeck, U. Schroeder, T. Mikolajick, On the stabilization of ferroelectric negative capacitance in nanoscale devices. Nanoscale 10,10891-10899 (2018). doi:10.1039/C8NR02752H Medline

65. H. Mulaosmanovic et al., in 2015 IEEE International Electron Devices Meeting (IEDM). (IEEE, 2015), pp. 26.28. 21-26.28. 23.

66. H. Mulaosmanovic, T. Mikolajick, S. Slesazeck, Accumulative polarization reversal in nanoscale ferroelectric transistors. ACS Appl. Mater. Interfaces 10, 2399724002 (2018). doi:10.1021/acsami.8b08967 Medline

67. H. Mändar, R. Rammula, A. Aidla, J. Aarik, Atomic layer deposition of epitaxial $\mathrm{HfO}_{2}$ thin films on r-cut sapphire. J. Mater. Res. 28, 1680-1686 (2013). doi:10.1557/imr.2013.120

68. A. Rosental, A. Tarre, A. Gerst, J. Sundqvist, A. Hårsta, A. Aidla, J. Aarik, V. Sammelselg, T. Uustare, Gas sensing properties of epitaxial $\mathrm{SnO}_{2}$ thin films prepared by atomic layer deposition. Sens. Actuators B Chem. 93, 552-555 (2003). doi:10.1016/S0925-4005(03)00236-3

69. H. Kim, C. O. Chui, K. C. Saraswat, P. C. McIntyre, Local epitaxial growth of $\mathrm{ZrO}_{2}$ on Ge (100) substrates by atomic layer epitaxy. Appl. Phys. Lett. 83, 2647-2649 (2003). doi:10.1063/1.1613031

70. K. Klepper, O. Nilsen, H. Fjellvåg, Epitaxial growth of cobalt oxide by atomic layer deposition. J. Cryst. Growth 307, 457-465 (2007). doi:10.1016/j.jcrysgro.2007.06.028

\section{ACKNOWLEDGMENTS}

We thank Nicola Spaldin for critical and constructive comments on the manuscript. JHL appreciates Youngkuk Kim, Hyunah Kang, Chang Hoon Kim and Heung-Sik Kim for useful discussions. Funding: The work on memories is mainly supported by Samsung Research Funding and Incubation Center of Samsung Electronics under Project Number SRFC-MA1902-01. The work on scale-free ferroelectricity is supported by the Creative Materials Discovery (2017M3D1A1040828), the MOTIE (Ministry of Trade, Industry Energy) (No. 10080657), KRSC (Korea Semiconductor Research Consortium) program, Basic Research Laboratory (NRF2017R1A4A1015323), and the Research Project Funded by U-K Brand (1.200033.01, 1.200030.01). UW thanks UNIST for hospitality and funding from a JC Bose National Fellowship of SERB, Government of India and support from the Department of Science and Technology for the India-Korea Joint Network Center in computational materials science. We appreciate Supercomputing Center/Korea Institute of Science and Technology Information for supercomputing resources including technical support (KSC-2020-CRE-0088). Author contributions: JHL conceived the idea and supervised the work. H.-J.L., Y.K., U.W., and J.H.L. designed and carried out DFT calculations, and M.L., H.Y., and J.J. helped analyzing the data. K.L. and S.C.C. provided experimental verifications. J.H.L., U.W. and H.-J.L. developed the theory and wrote the manuscript. Competing interests: J.H.L. and H.-J.L. are inventors on KR patent application (10-2020-0047206) submitted by the Ulsan National Institute of Science and Technology (UNIST) that covers ultimately-high-density ferroelectric memories and deterministic multi-level devices induced by flat phonon bands. Data and materials availability: All data are available in the main text or the supplementary materials.

\section{SUPPLEMENTARY MATERIALS}

science.sciencemag.org/cgi/content/full/science.aba0067/DC1

Materials and Methods

Supplementary Text S1 to S3

Figs. S1 to S15

References (31-70)

28 October 2019; resubmitted 29 April 2020

Accepted 16 June 2020

Published online 2 July 2020

10.1126/science.aba0067 
(a)

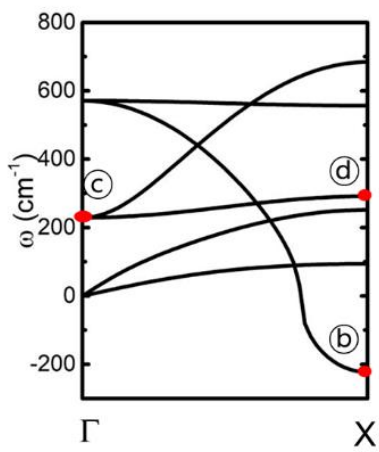

(b)

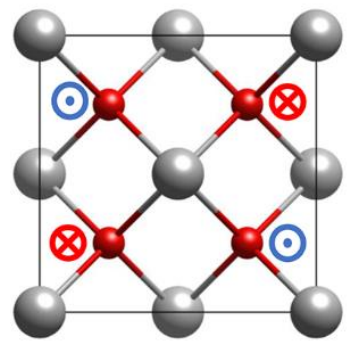

(c)

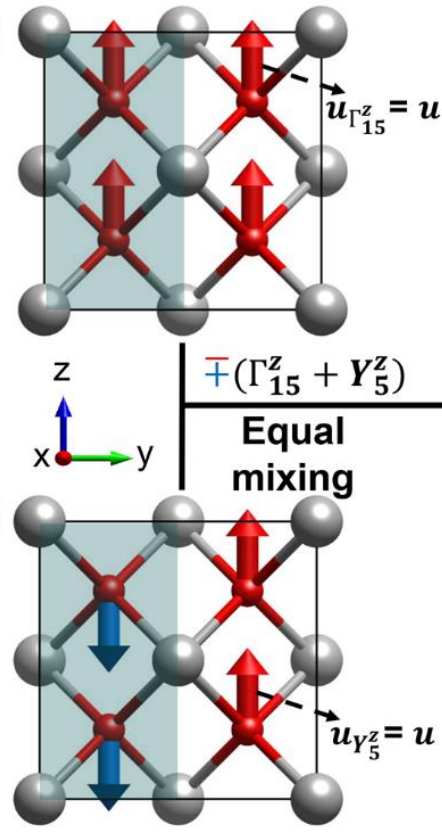

Cubic

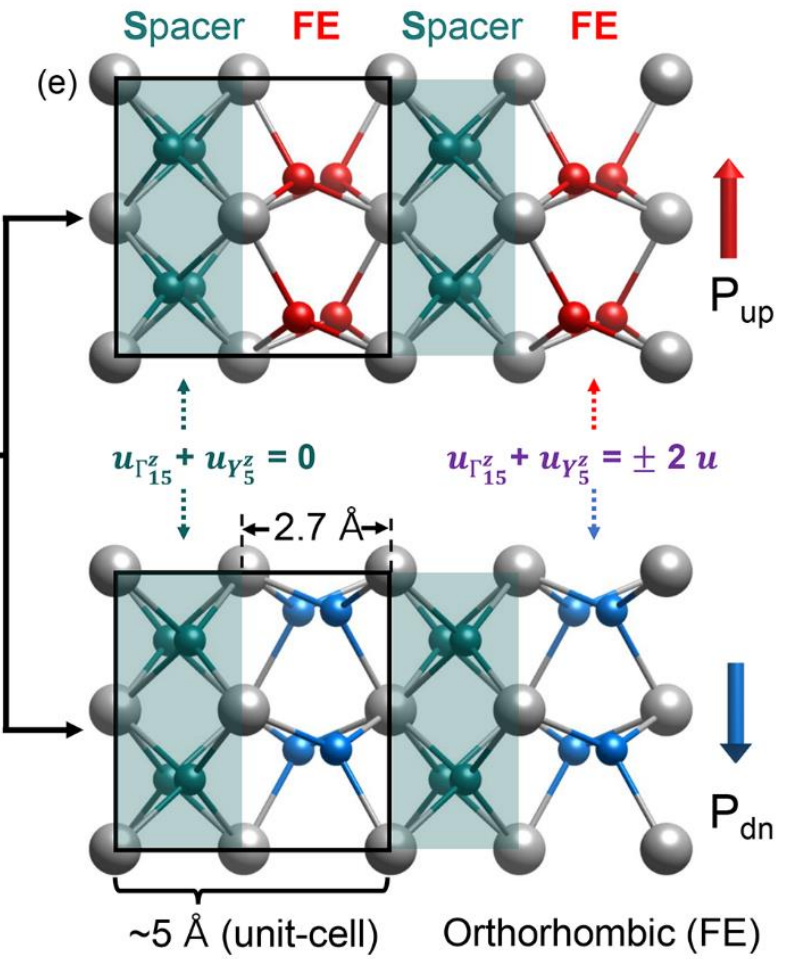

Fig. 1. Structural origin of alternating ferroelectric and nonpolar layers in orthorhombic $\mathrm{HfO}_{2}$. (A) Phonon dispersion of the cubic phase; each red dot labeled b, c, and d denotes primary instability of (B) $\mathrm{X}_{2}^{\prime}$ mode, (C) $\Gamma_{15}{ }^{z}$, and (D) $Y_{5}{ }^{z}$, where arrows denote $u$, the displacements of oxygen atoms. (E) Polar $\Gamma_{15}{ }^{z}$ and anti-polar $Y_{5}{ }^{z}$ phonons condense in-phase with equal magnitude to generate orthorhombic structure consisting of alternating spacer layer and ferroelectric layer with up (top), and down (bottom) polarization, respectively. Silver balls denote $\mathrm{Hf}$ atoms, red and blue balls denote oxygen atoms in ferroelectric layer with up and down polarization, respectively, and green balls indicate oxygen atoms belonging to the spacer layer. 
(a)
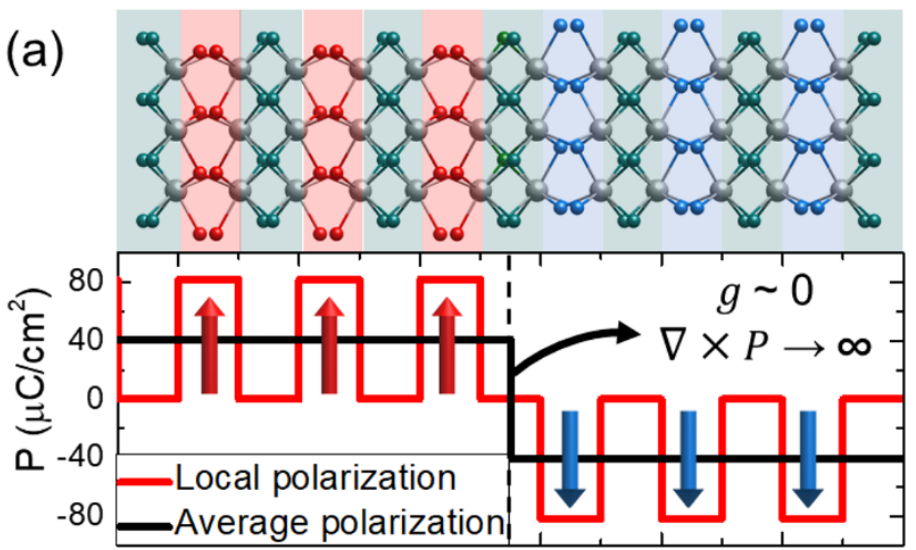

$\mathrm{HfO}_{2}$ Zero width domain wall

$$
\varepsilon_{\mathrm{x}}=-0.17 \%, \varepsilon_{\mathrm{y}}=0.12 \%, \varepsilon_{\mathrm{z}}=0 \%
$$

(c)
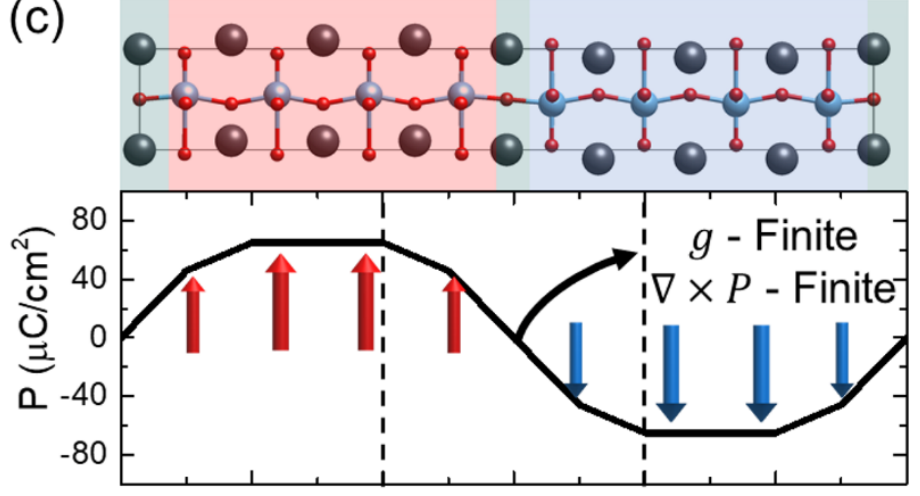

$\mathrm{PbTiO}_{3} \quad$ Finite domain wall

$\varepsilon_{\mathrm{x}}=0.68 \%, \varepsilon_{\mathrm{y}}=4.54 \%, \varepsilon_{\mathrm{z}}=-4.83 \%$ (b)

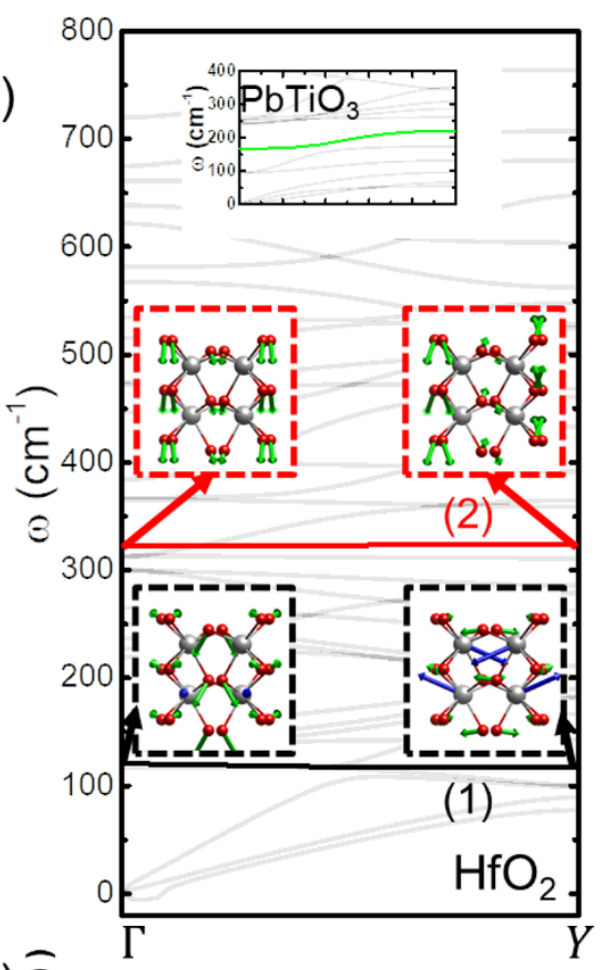

(d) $\overline{\mathrm{a}}$

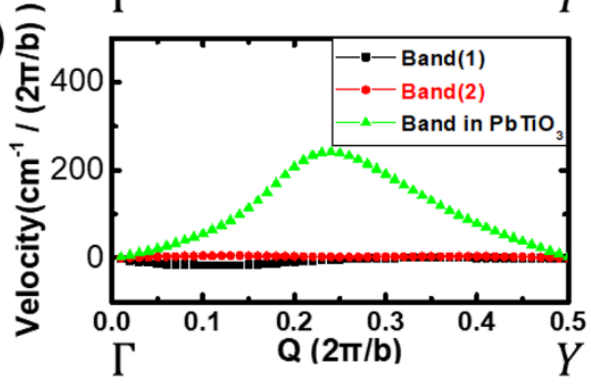

Fig. 2. Flat bands and zero-width domain wall in $\mathrm{HfO}_{2}$, contrary to the diffused domain wall in $\mathrm{PbTiO}_{3}$. $(\mathrm{A}$ and C) Atomic structure of the domain wall and variation in local polarization along the direction perpendicular to the domain walls in $(\mathrm{A}) \mathrm{HfO}_{2}$ and $(\mathrm{C}) \mathrm{PbTiO}_{3}$. Red and black lines correspond to the local polarization averaged over their half and single unit cells, respectively. While $\left(\vec{\nabla} \times \vec{P}=\frac{\partial P_{z}}{\partial y}\right)$ is small and spreads over a few unit cells away from the domain wall in $\mathrm{PbTiO}_{3}$, it is singularly large and highly localized at the domain wall in $\mathrm{HfO}_{2}$. (B) This is due to its low energy cost $(g \sim 0)$ guaranteed by the flatness of polar phonon bands involving the polar and anti-polar modes condensed in orthorhombic $\mathrm{HfO}_{2}$. Flat polar bands are marked with black and red lines and eigenmodes at $\mathrm{T}$ and $\mathrm{Y}$ in the bands are depicted in insets. This flatness of the bands in $\mathrm{HfO}_{2}$ is in sharp contrast to a dispersive band in $\mathrm{PbTiO}_{3}$ [(B), top, inset]. (D) Phonon velocities of the flat bands in $\mathrm{HfO}_{2}$ are nearly zero, while that of polar band in $\mathrm{PbTiO}_{3}$ has a finite value. For $\mathrm{PbTiO}_{3}$, black balls denote $\mathrm{Pb}$ atoms, and sky-blue and red balls denote Ti and oxygen atoms, respectively. 


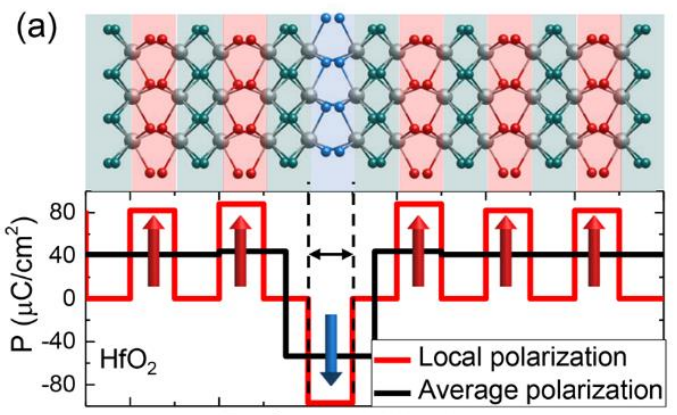

Perfect stabilization

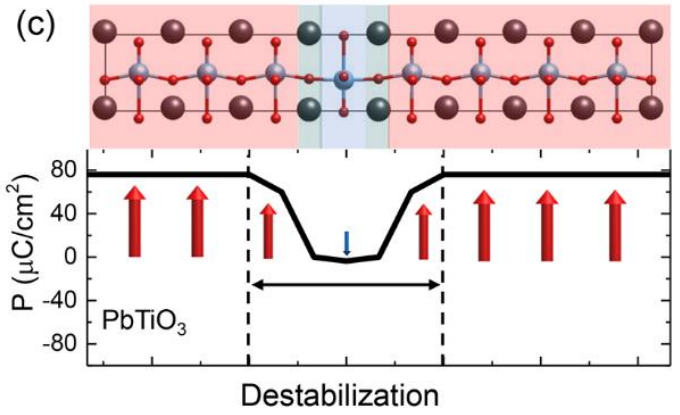

(b)

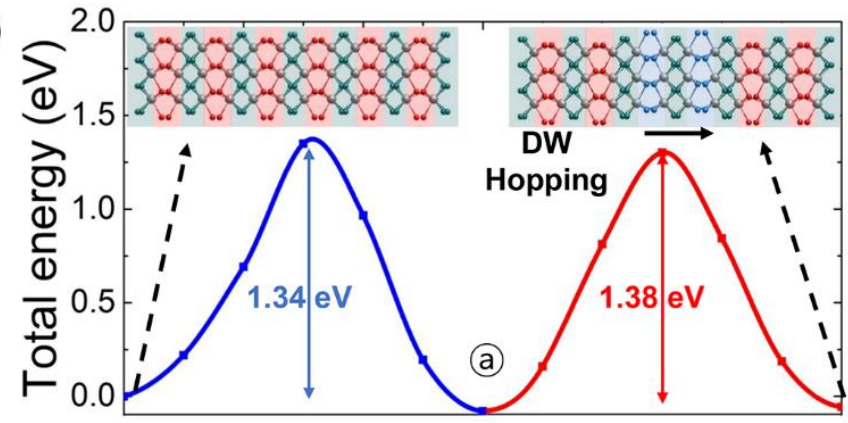

(d)

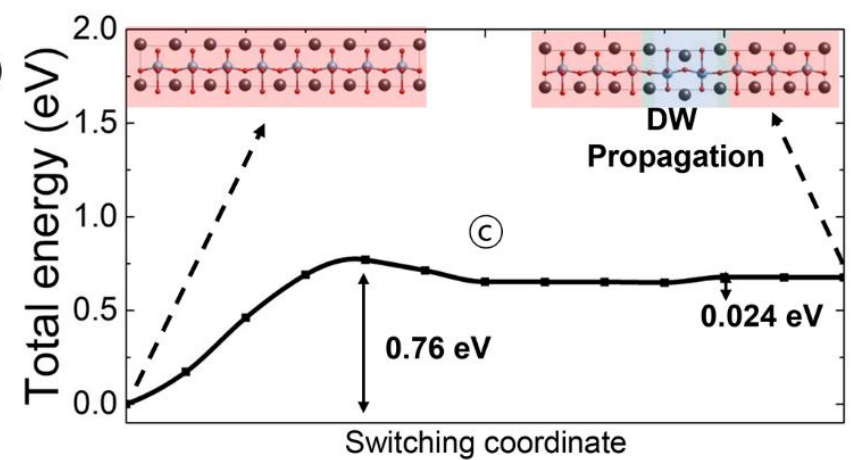

Fig. 3. Robust stability of a half-unit-cell-width ferroelectric domain. (A) Atomic structure of the thinnest domain and variation in polarization along the direction perpendicular to the domain walls in $\mathrm{HfO}_{2}$. (B) Energy along the path of polarization switching of $\mathrm{HfO}_{2}$ starting from the uniformly polarized structure $[(B)$, left inset] to a state with reversed polarization in two layers [(B), right inset] passing through the state in (A). (C) In contrast to the domain polarization that is unsuppressed relative to bulk $P$ in $\mathrm{HfO}_{2}(A)$, it is substantially suppressed inside the switched domain as well as a few unit cells away from the domain wall of $\mathrm{PbTiO}_{3}$. (D) Remarkably, the robust stability of a half-unit-cell-wide domain of $\mathrm{HfO}_{2}(\mathrm{~B})$ is in complete contrast with the marginal stability of single-unit-cell domain in $\mathrm{PbTiO}_{3}$ which spontaneously expands to wider domains. Successive switching in the next layer [(B), red line] of $\mathrm{HfO}_{2}$, has an energy barrier comparable to the first switching process [(B), blue line] in $\mathrm{HfO}_{2}$, in contrast to a small energy barrier of successive switching in $\mathrm{PbTiO}_{3}(\mathrm{D})$. 
(a)

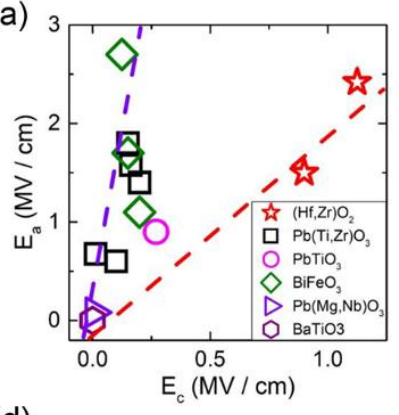

(d)

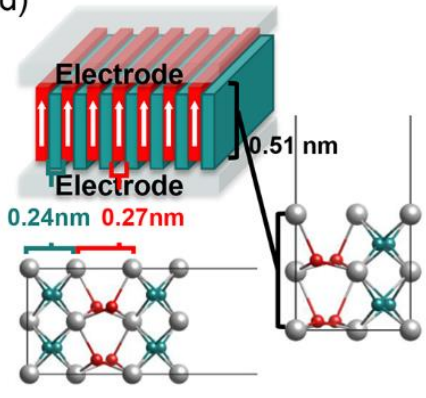

(b)
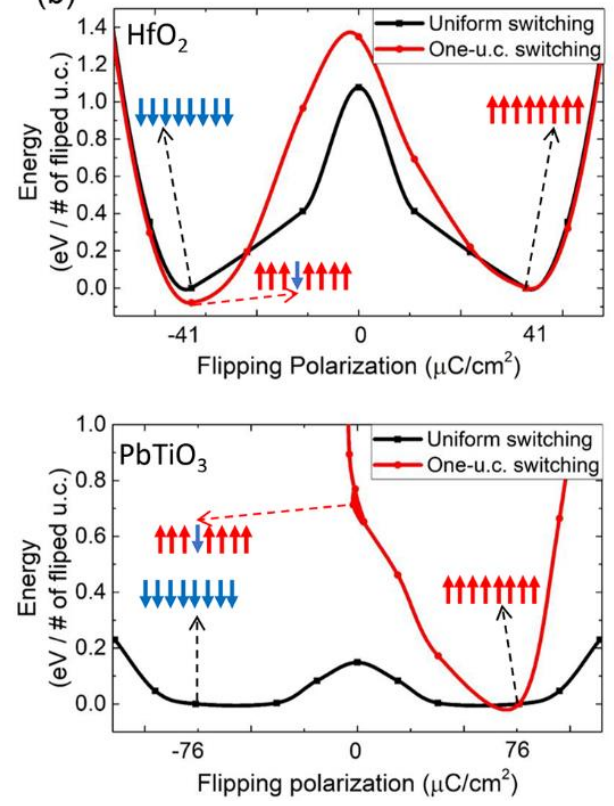

(c)
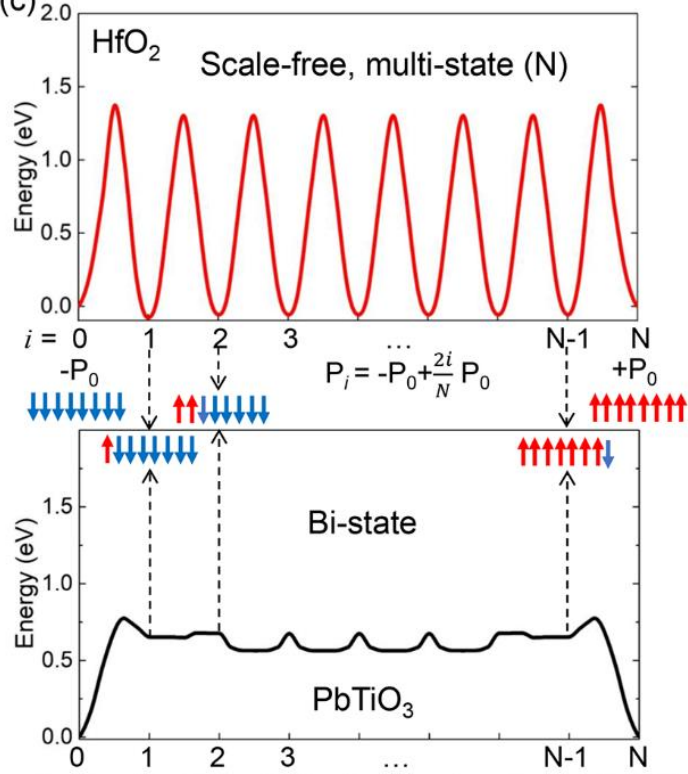

Number $(i)$ of flipped $2 \mathrm{D}$ polar domains of unit-cell-width

Fig. 4. Experimental activation and coercive fields revealing unusual switching behavior of $\mathrm{HfO}_{2}$ and simulated energetics demonstrating its perfectly scale-free 2D domain switching of $0.27 \mathrm{~nm}$ width. (A) Experimental values of activation $\left(E_{\mathrm{a}}\right)$ and coercive $\left(E_{\mathrm{c}}\right)$ fields for various ferroelectrics $\left[\mathrm{BaTiO}_{3},(16)\right.$; $\left.\mathrm{Pb}(\mathrm{Zr}, \mathrm{Ti}) \mathrm{O}_{3},(17,18) ; \mathrm{BiFeO}_{3},(19,21) ; \mathrm{Pb}(\mathrm{MgNb}) \mathrm{O}_{3},(20) ; \mathrm{PbTiO}_{3},(21) ;(\mathrm{Hf}, \mathrm{Zr}) \mathrm{O}_{2},(5,6)\right]$. While $E_{\mathrm{c}}$ is 10 times smaller than $E_{\mathrm{a}}$ in conventional ferroelectrics, these are comparable in $\mathrm{HZO}$, implying that collective and individual domain switching occur at the same field. (B) Calculated energy curves along paths of switching uniform and local (one-unit-cell) polarization in $\mathrm{HfO}_{2}$ (top) and $\mathrm{PbTiO}_{3}$ (bottom). (C) Energy of unit-cell-by-unitcell width switching: while switching of local polarization in consecutive unit cells in $\mathrm{PbTiO}_{3}$ shows bi-stable $\left( \pm P_{0}\right)$ behavior (bottom), domains of any width in $\mathrm{HfO}_{2}$ are equally stable with the same energy barrier of switching (top) showing its multi-state and scale-free ferroelectric behavior in stability and switchability. (D) Schematic of $\mathrm{HfO}_{2}$-based capacitive memory device with ultrahigh density because the improper ferroelectricity allows no critical thickness, as confirmed with first-principles calculations [(D), right] and there is no critical lateral width of ferroelectricity in $\mathrm{HfO}_{2}[(\mathrm{D})$, bottom] due to the presence of spacer layers. 